\title{
Correlation between Correlations: Process and Time in Quantum Networks
}

\author{
Günter Mahler and Ilki Kim \\ Institut für Theoretische Physik I, Universität Stuttgart \\ Pfaffenwaldring 57, 70550 Stuttgart, Germany \\ email: mahler@theo.physik.uni-stuttgart.de
}

\begin{abstract}
We study a special inhomogeneous quantum network consisting of a ring of $M$ pseudo-spins (here $M=4$ ) sequentially coupled to one and the same central spin under the influence of given pulse sequences (quantum gate operations). This architecture could be visualized as a quantum Turing machine with a cyclic "tape". Rather than input-output-relations we investigate the resulting process, i.e. the correlation between one- and two-point expectation values ("correlations") over various timesteps. The resulting spatio-temporal pattern exhibits many non-classical features including Zeno-effects, violation of temporal Bell-inequalities, and quantum parallelism. Due to the strange web of correlations being built-up, specific measurement outcomes for the tape may refer to one or several preparation histories of the head. Specific families of correlation functions are more stable with respect to dissipation than the total wave-function.
\end{abstract}

\section{Introduction}

It has been shown that certain computational problems scale more favorably when carried out on a quantum system than on any classical computer (see, e.g., [1]). The underlying "quantum complexity" may thus reduce computational complexity. Architectures for abstract quantum networks appropriate for such potential applications have been discussed (cf., e.g., [2]).

On the other hand, the control of such quantum networks appears to scale very badly with system size: In fact, in the language of statistical physics, that control would amount to use "micro-states" rather than "macro-states", a challenging undertaking, indeed. It should therefore not come as a surprise that virtually all proposals up to now face severe problems when trying to go beyond the (coherent) control of something like $N=10$ pseudospins [3, 4, 5, 6]. Also the detailed theoretical simulation will become increasingly difficult if not eventually impossible beyond that limit. Fortunately, as will be shown below, even such small networks may show a surprisingly rich behavior in terms of correlation-functions. Rather than the entanglement as such this pattern of correlations should be considered as the basis of the expected computational efficiency as well as other potential applications.

\section{Composite systems}

\section{$2.1 \quad$ States}

The system we are going to investigate here is composed of $M+1$ spins, $\mu=S, 1,2, \cdots, M$. The respective states are $\mid p(\mu)>, p=0,1$. The corresponding product basis is $\mid u(M) \cdots$ 
$r(2) q(1) p(S)>\equiv \mid u \cdots r q p>$. Arranged in the order of increasing binary numbers we also introduce the single-index notation $\mid s>, s=0,1, \cdots, 2^{M+1}-1$ by identifying $\mid 0>=$ $|0 \cdots 000>| 1>,=|0 \cdots 001>| 2>,=\mid 0 \cdots 010>$, etc. This single-index representation will not only serve as a means to simplify some algebra. It reminds us that one can entirely avoid talking about entanglement while, nevertheless, keeping the product-space background still operative, though in a more subtle way: In terms of the specific operator combinations and their expectation values.

\subsection{Cluster-operators}

For $M+1=5$ there are $\left(2^{5}\right)^{2}=1024$ orthogonal basis operators. One possible choice would be products of local transition-operators, $\hat{P}_{p q}(\mu)=|p(\mu)><q(\mu)|$. For reasons that will become clear shortly it is more convenient to separate out the local unit operators $\hat{1}(\mu)$ so that the remaining operators become traceless. Such a scheme is provided by the Hermitian and unitary $S U(2)$-generators, $\hat{\lambda}_{j}(\mu)$,

$$
\begin{aligned}
& \hat{\lambda}_{1}(\mu)=\hat{P}_{01}(\mu)+\hat{P}_{10}(\mu) \\
& \hat{\lambda}_{2}(\mu)=i \hat{P}_{01}(\mu)-i \hat{P}_{10}(\mu) \\
& \hat{\lambda}_{3}(\mu)=\hat{P}_{11}(\mu)-\hat{P}_{00}(\mu) \\
& \hat{\lambda}_{0}(\mu)=\hat{P}_{11}(\mu)+\hat{P}_{00}(\mu)=\hat{1}(\mu) .
\end{aligned}
$$

The corresponding product operators $(j, k, l, m, n=0,1,2,3)$ [7]

$$
\hat{Q}_{j k l m n}=\hat{\lambda}_{j}(S) \hat{\lambda}_{k}(1) \hat{\lambda}_{l}(2) \hat{\lambda}_{m}(3) \hat{\lambda}_{n}(4)
$$

with $\left(\hat{Q}_{j k l m n}\right)^{2}=\hat{1}$ for any $(j, k, l, m, n)$ and

$$
\operatorname{Tr}\left\{\hat{Q}_{j k l m n} \hat{Q}_{j^{\prime} k^{\prime} l^{\prime} m^{\prime} n^{\prime}}\right\}=2^{5} \delta_{j j^{\prime}} \delta_{k k^{\prime}} \delta_{l l^{\prime}} \delta_{m m^{\prime}} \delta_{n n^{\prime}}
$$

then come in 6 classes, depending on the number $c=0,1, \cdots, M+1$ of subsystems they act on, i.e. the number of indices unequal zero. $\hat{Q}_{00000}=\hat{1}$ is the only $c=0$ cluster operator. When transcribed to the single index-space, $s=0,1, \cdots, 2^{5}-1$, these operators appear like a set of "generalized" $S U(2)$-operators of the form given in eq. (1) with each single transition or projection operator replaced by a group of $2^{M}=16$. Such operator combinations would be hard if not impossible to implement in a simple one-particle system with $2^{5}$ states; they reflect the structure of the underlying product space. Correspondingly, the expectation-value of any cluster-operator is a sum of $2^{M+1}$ density matrix elements in the single-index space. Examples for $M=2$ are the $c=1$-cluster operators,

$$
\begin{aligned}
& \hat{Q}_{300}=\left(\hat{P}_{11}+\hat{P}_{33}+\hat{P}_{55}+\hat{P}_{77}\right)-\left(\hat{P}_{00}+\hat{P}_{22}+\hat{P}_{44}+\hat{P}_{66}\right) \\
& \hat{Q}_{030}=\left(\hat{P}_{22}+\hat{P}_{33}+\hat{P}_{66}+\hat{P}_{77}\right)-\left(\hat{P}_{00}+\hat{P}_{11}+\hat{P}_{44}+\hat{P}_{55}\right)
\end{aligned}
$$

or $c=2$-cluster operators such as,

$$
\begin{aligned}
& \hat{Q}_{330}=\left(\hat{P}_{00}+\hat{P}_{33}+\hat{P}_{44}+\hat{P}_{77}\right)-\left(\hat{P}_{11}+\hat{P}_{22}+\hat{P}_{55}+\hat{P}_{66}\right) \\
& \hat{Q}_{303}=\left(\hat{P}_{00}+\hat{P}_{22}+\hat{P}_{55}+\hat{P}_{77}\right)-\left(\hat{P}_{11}+\hat{P}_{33}+\hat{P}_{44}+\hat{P}_{66}\right) .
\end{aligned}
$$


Any operator $\hat{A}$ in the $2^{5}$-dimensional Hilbert-space of spin-states can be represented as (summation over repeated indices),

$$
\hat{A}=\frac{1}{2^{5}} A_{j k l m n} \hat{Q}_{j k l m n}
$$

with the parameters

$$
A_{j k l m n}=\operatorname{Tr}\left\{\hat{A} \hat{Q}_{j k l m n}\right\}
$$

(Tr means trace over the total Hilbert-space.) In particular, the network-Hamiltonian $\hat{H}$ can be specified by the model parameters $H_{j k l m n}$; they are usually constrained to $c=0,1$ and 2-cluster-terms [7]. The density operator $\hat{\rho}$ is uniquely defined by the set of expectationvalues (note that $\hat{Q}_{j k l m n}$ is unitary)

$$
-1 \leq K_{j k l m n}=\operatorname{Tr}\left\{\hat{\rho} \hat{Q}_{j k l m n}\right\} \leq 1
$$

with $c$-cluster-operators defining $c$-particle correlations. For a pure-state, $\hat{\rho}=|\psi><\psi|$, eq. (8) reduces to

$$
K_{j k l m n}=<\psi\left|\hat{Q}_{j k l m n}\right| \psi>.
$$

By definition, $K_{00000}=1$; the local Bloch-vectors $K_{j 0000}, K_{0 k 000}, K_{00 l 00}$, etc. $(j, k, l=1,2,3)$ are equivalent to the respective reduced density matrices. A pure local state has Blochvector-length 1. For so-called product-states all these correlations factor into one-point functions, i.e. $K_{j k l m n}=K_{j 0000} K_{0 k 000} K_{00 l 00} K_{000 m 0} K_{0000 n}$, but, in general, they are independent. Local realism (cf. [8]), to be sure, postulates that an appropriate distribution of local variables (eigenvalues $\lambda_{j}= \pm 1$ ) could explain all these correlation functions rendering them statistically dependent; at least for larger networks this approach is no longer tenable. On the other hand, as will be shown below, the quantum mechanical evolution generates "correlations between correlations".

For later reference we also define symmetrized correlation-functions within one and the same system $\mu$ :

$$
C_{A B}^{(\mu)}=\frac{1}{2}(\operatorname{Tr}\{\hat{\rho} \hat{A}(\mu) \hat{B}(\mu)\}+\operatorname{Tr}\{\hat{\rho} \hat{B}(\mu) \hat{A}(\mu)\}) .
$$

Restricting ourselves to traceless operators, this correlation is independent of $\hat{\rho}$ (for twodimensional Hilbert-spaces) and can simply be written as the normalized scalar product between the two representing vectors [7]; for $\mu=S$, e.g.,

$$
C_{A B}^{(S)}=\frac{1}{2^{10}} A_{j 0000} B_{j 0000}
$$

\subsection{Unitary transformations}

A unitary transformation of an operator $\hat{A}$,

$$
\hat{A}^{\prime}=\hat{U} \hat{A} \hat{U}^{+}
$$

with $\hat{U}^{+} \hat{U}=\hat{U} \hat{U}^{+}=\hat{1}$, reads in terms of the $S U(2)$ - parameters,

$$
A_{j k l m n}^{\prime}=X_{j k l m n}^{j^{\prime} k^{\prime} l^{\prime} m^{\prime} n^{\prime}} A_{j^{\prime} k^{\prime} l^{\prime} m^{\prime} n^{\prime}}
$$


where

$$
X_{j k l m n}^{j^{\prime} k^{\prime} l^{\prime} m^{\prime} n^{\prime}}=\frac{1}{2^{5}} \operatorname{Tr}\left\{\hat{U}^{+} \hat{Q}_{j k l m n} \hat{U} \hat{Q}_{j^{\prime} k^{\prime} l^{\prime} m^{\prime} n^{\prime}}\right\}
$$

(For $\hat{U}=\hat{1}, X$ is just the unit matrix, see eq. (3)). There are different types: We may distinguish transformations which operate in certain subspaces only. The locally selective transformation $\hat{U}(S)$ in the $n=2$-dimensional local Hilbert-space of $S$, e.g., is equivalent to a local rotation of the $S U(2)$-parameters with respect to the first index $j$, generated by (cf. [9])

$$
\begin{aligned}
X_{j k l m n}^{j^{\prime} k^{\prime} l^{\prime} m^{\prime} n^{\prime}} & =X_{j j^{\prime}}^{(S)} \delta_{k k^{\prime}} \delta_{l l^{\prime}} \delta_{m m^{\prime}} \delta_{n n^{\prime}} \\
X_{j j^{\prime}}^{(S)} & =\frac{1}{2} \operatorname{Tr}_{S}\left\{\hat{U}^{+}(S) \hat{\lambda}_{j}(S) \hat{U}(S) \hat{\lambda}_{j^{\prime}}(S)\right\}
\end{aligned}
$$

(Here, $\operatorname{Tr}_{S}$ means trace over the subspace of $S$ only.) As $X_{00}^{(S)}=1, X_{j j^{\prime}}^{(S)}=0$ if either $j$ or $j^{\prime}$ is zero, all parameters $A_{0 k l m n}$ are invariants. Correspondingly, a unitary transformation $\hat{U}(S, 1)$ leaves the expectation values $A_{00 l m n}$ unchanged, etc. These invariants (conservation laws) are important characteristics of the respective transformations.

\subsection{Time}

As we do not consider equations of motion explicitly, time enters at most indirectly: To specify change, order and duration. For closed systems, unitary transformations are the only allowed type of changes (of states or observables) in closed quantum systems. Typically they are generated by the underlying Hamilton model. In the Schrödinger-picture this unitary transformation is applied to $\hat{\rho}$, in the Heisenberg-picture the inverse transformation (replacing $\hat{U}$ by $\hat{U}^{+}$and vice versa) is applied to the observables.

Parameter time $T$ will come in with respect to the order, in which certain transformations are applied, as a continuous parameter controlling the individual transformation quantitatively ("pulse length" $t$ ), and, eventually, with respect to the order of measurements. Finally, the induced dynamics can be characterized by correlation- and recurrence-times.

\section{The Turing model}

Our system is sketched in Fig. 11: Spin $S$ is the "Turing head", the other $\mu=1,2 \cdots, M=4$ subsystems denote memories (as part of a circular "Turing tape"); the latter do not interact directly and are separated by "empty" cells. The head interacts with at most one cell at a time [10]; it moves clockwise and step by step to one of the $2 M$ positions on the tape; there is no need for a feedback between the internal quantum state of the network and this pre-determined "classical" movement.

We assume to have explicit control over the model parameters $H_{j k l m n}$ defining the Hamiltonian, which may even be modified in terms of pulses in parameter-time $\left(t_{j}\right.$ is the pulse-length):

$$
\hat{H}(t)=\hat{H}_{j} \text { for } T_{j-1} \leq t<T_{j-1}+t_{j} \equiv T_{j} .
$$

Granted this access we can implement virtually any unitary transformation via

$$
\hat{U}\left(T_{j-1}+t_{j}, T_{j-1}\right) \equiv \hat{U}_{j}=e^{-i \hat{H} t_{j} / \hbar}
$$


( $j$ is the step number), though this may seriously be limited in practice. If the Turing head is over an empty cell (tape position $2 \mu-1$ ), a local transformation $\hat{U}_{\alpha}(S)$ on $S$ is applied, if it is in contact with a memory cell $\mu$ at position $2 \mu$ a pair transformation on $(S, \mu)$ is induced $(\mu=1,2 \cdots, M)$.

\subsection{Local transformation on $(\mathrm{S})$}

Let us consider the one-parameter-form

$$
\begin{aligned}
& |0(S)>\longrightarrow \cos (\alpha / 2)| 0(S)>-i \sin (\alpha / 2) \mid 1(S)> \\
& |1(S)>\longrightarrow-i \sin (\alpha / 2)| 0(S)>+\cos (\alpha / 2) \mid 1(S)>
\end{aligned}
$$

which can be generated by $(M=4)$

$$
\hat{U}_{\alpha}(S)=\hat{Q}_{00000} \cos (\alpha / 2)-\hat{Q}_{10000} i \sin (\alpha / 2)=\hat{U}_{-\alpha}^{+}(S) .
$$

According to eqs. (19), (15) and (2), we find

$$
\begin{aligned}
X_{j j^{\prime}}^{(S)=} & \cos ^{2}(\alpha / 2) \delta_{j j^{\prime}}+\frac{1}{2} \sin ^{2}(\alpha / 2) \operatorname{Tr}_{S}\left\{\hat{\lambda}_{1} \hat{\lambda}_{j} \hat{\lambda}_{1} \hat{\lambda}_{j^{\prime}}\right\} \\
& +\frac{i}{4} \sin \alpha \operatorname{Tr}_{S}\left\{\hat{\lambda}_{1} \hat{\lambda}_{j} \hat{\lambda}_{j^{\prime}}-\hat{\lambda}_{j} \hat{\lambda}_{1} \hat{\lambda}_{j^{\prime}}\right\}
\end{aligned}
$$

so that $X_{00}^{(S)}=X_{11}^{(S)}=1, X_{22}^{(S)}=X_{33}^{(S)}=\cos \alpha, X_{32}^{(S)}=-X_{23}^{(S)}=\sin \alpha$. (Here and in the following all terms not explicitly given are zero.) This matrix $X_{i j}^{(S)}$ defines a rotation of the Bloch-vector of $S$ around the $k=1$-axis in the 2,3-plane. The phase $\alpha$ may be taken to result from a pulse of duration $t$

$$
\alpha=g t
$$

where $g$ would be the coupling strength to an external optical driving field. The correlation function between $\hat{A}=\hat{\lambda}_{3}(S)$ transformed by $\phi$ and the same operator transformed by phase angle $\phi+\alpha$ then is, according to eq. (11),

$$
C_{33}^{(S)}(\phi, \phi+\alpha)=\cos \alpha \text {. }
$$

Based on eq. (21) this expectation value can be interpreted as a 2-time 1-particle correlation function in the Heisenberg-picture. Combinations of these have been shown to violate temporal Bell inequalities [11].

\subsection{Pair transformation on $(S, \mu)$}

This unitary transformation is taken as the conditioned $\pi$-pulse, $(q=0,1)$

$$
\begin{array}{c|c|c|}
\text { Resonance: } & |0(S) 0(\mu)>\longleftrightarrow| 0(S) 1(\mu)> \\
\text { Off-resonance: } & |1(S) q(\mu)>\longleftrightarrow| 1(S) q(\mu)>
\end{array}
$$

which we may write, in terms of cluster operators, in the form

$$
\begin{aligned}
\hat{U}(S, 1) & =\hat{P}_{00}(S) \hat{\lambda}_{1}(1)+\hat{P}_{11}(S) \hat{1}(1) \\
& =\frac{1}{2}\left(\hat{Q}_{00000}+\hat{Q}_{30000}+\hat{Q}_{01000}-\hat{Q}_{31000}\right)=\hat{U}^{+}(S, 1) .
\end{aligned}
$$


These operators $\hat{U}(S, \mu)$ commute; their implementation requires pair interactions, which make the transition frequency in subsystem $\mu$ depend on the state of subsystem $S$ [7] [12]. This transformation has become known as the (quantum-) controlled NOT [2], as subsystem $S$ acts as a control for a $\pi$-pulse on $\mu$. We may associate a fixed pulse duration $t_{0}$ with this implementation; here we assume $t_{0} \approx 0$. In general, the two types of unitary operators do not commute:

$$
\left[\hat{U}(S, \mu), \hat{U}_{\alpha}(S)\right]=\sin (\alpha / 2)\left(\hat{1}(\mu)-\hat{\lambda}_{1}(\mu)\right) \hat{\lambda}_{2}(S)
$$

\section{The process}

\subsection{The first cycle}

We are now in a position to follow up the ordered sequence of $2 M=8$ unitary transformations,

$$
\left|\psi^{(1, j)}>=\hat{U}_{j}\right| \psi^{(1, j-1)}>
$$

where $(\mu=1,2, \cdots, M)$

$$
\begin{aligned}
\hat{U}_{2 \mu-1} & =\hat{U}_{\alpha_{\mu}}(S) \\
\hat{U}_{2 \mu} & =\hat{U}(S, \mu) .
\end{aligned}
$$

Here and in the following the upper index pair in parenthesis denotes the cycle number $m$ and the step number $j$, respectively. With $j=2 \mu(\mu=1,2, \cdots, M)$ we may associate the time (cf. eq. (21))

$$
T_{2 \mu}=\sum_{i=1}^{\mu} t_{2 i-1}=\sum_{i=1}^{\mu} \alpha_{i} / g \approx T_{2 \mu-1} .
$$

$T_{2 M}$ is then the time needed for each cycle. Now, let the initial state be $\left|\psi^{(1,0)}>=\right| 0>=$ |00000 > so that the local Bloch-vectors are given by

$$
K_{30000}^{(1,0)}=K_{03000}^{(1,0)}=\cdots=K_{00003}^{(1,0)}=-1 .
$$

In the first step we apply the local transformation with a phase $\alpha_{1}$ leading to

$$
\left|\psi^{(1,1)}>=\cos \left(\alpha_{1} / 2\right)\right| 0>-i \sin \left(\alpha_{1} / 2\right) \mid 1>.
$$

In the second step we execute the pair transformation on $(S, 1)$ :

$$
\left|\psi^{(1,2)}>=\cos \left(\alpha_{1} / 2\right)\right| 2>-i \sin \left(\alpha_{1} / 2\right) \mid 1>\text {. }
$$

In the third step we again apply the local transformation, now with phase $\alpha_{2}$, leading to

$$
\begin{aligned}
\mid \psi^{(1,3)}>= & \cos \left(\alpha_{1} / 2\right) \cos \left(\alpha_{2} / 2\right)\left|2>-i \cos \left(\alpha_{1} / 2\right) \sin \left(\alpha_{2} / 2\right)\right| 3> \\
& -i \sin \left(\alpha_{1} / 2\right) \cos \left(\alpha_{2} / 2\right)\left|1>-\sin \left(\alpha_{1} / 2\right) \sin \left(\alpha_{2} / 2\right)\right| 0>.
\end{aligned}
$$

In the "Heisenberg-picture", this implies between step 2 and step 3 the local correlation as given by eq. (22) with $\alpha=\alpha_{2}$. In the 4 th step the pair transformation on $(S, 2)$ implies

$$
\begin{aligned}
\mid \psi^{(1,4)}>= & \cos \left(\alpha_{1} / 2\right) \cos \left(\alpha_{2} / 2\right)\left|6>-i \cos \left(\alpha_{1} / 2\right) \sin \left(\alpha_{2} / 2\right)\right| 3> \\
& -i \sin \left(\alpha_{1} / 2\right) \cos \left(\alpha_{2} / 2\right)\left|1>-\sin \left(\alpha_{1} / 2\right) \sin \left(\alpha_{2} / 2\right)\right| 4>.
\end{aligned}
$$


This procedure is continued with respect to the next memory cells 3 and 4 (steps 5 through 8). We note that the single-subsystem expectation values of subsystem $S$ and $\mu$ obey the relations

$$
\begin{aligned}
& K_{30000}^{(1,2)}=-K_{03000}^{(1,2)}=K_{30000}^{(1,0)} \cos \alpha_{1} \\
& K_{30000}^{(1,4)}=-K_{03000}^{(1,4)}=K_{30000}^{(1,2)} \cos \alpha_{2} \text { etc. } \\
& K_{10000}^{(1,2 \mu)}=K_{20000}^{(1,2 \mu)}=0
\end{aligned}
$$

and as a consequence of the controlled-NOT-logic (cf. eq. (5)),

$$
K_{33000}^{(1,2)}=K_{30300}^{(1,4)}=K_{30030}^{(1,6)}=K_{30003}^{(1,8)}=-1 .
$$

We thus see that the two systems, $S$ and $\mu$, are strictly anti-correlated after step $2 \mu$ (the state $\left|\psi^{(1,2)}\right\rangle$, e.g., is actually an eigenstate of $\hat{Q}_{33000}$ !), while the local Bloch-vector-lengths are less than 1, i.e. local properties are not dispersion-free ("fuzzy"). This is typical for non-classical correlations. There can be strict correlations between fuzzy subsystems.

\subsection{Cycles $m \geq 1$.}

We can summarize and generalize the above results by introducing the following functions:

$$
\begin{aligned}
& \kappa^{(m, 2 M)}\left(\alpha_{1}, \alpha_{2} \cdots, \alpha_{j}\right)= \\
& \quad \frac{1}{2}\left[\cos \left(m \alpha_{1}\right) \cos \left(m \alpha_{2}\right) \cdots \cos \left(m \alpha_{j}\right)\right]+\frac{1}{2} \begin{cases}1 & m \text { even } \\
\cos \alpha_{1} \cos \alpha_{2} \cdots \cos \alpha_{j} & m \text { odd },\end{cases}
\end{aligned}
$$

$\kappa_{s}^{(m, 2 M)}$ as above with $\cos m \alpha_{1}$ replaced by $\sin m \alpha_{1}, \cos \alpha_{1}$ replaced by $-\sin \alpha_{1}$ and the 1 replaced by $0(j \leq M)$,

$$
\begin{aligned}
\phi_{k}^{(m, 2 M)}= & -\cos \left(m \alpha_{1} / 2\right) \cos \left(m \alpha_{2} / 2\right) \cdots \cos \left(m \alpha_{M} / 2\right) \\
\phi_{k}^{(m, 2 M)}= & \left.\cos \left((m+1) \alpha_{1} / 2\right) \cos \left((m+1) \alpha_{2} / 2\right) \cdots \cos \left((m+1) \alpha_{k} / 2\right)\right) \\
& \times \cos \left((m-1) \alpha_{k+1} / 2\right) \cdots \cos \left((m-1) \alpha_{M} / 2\right)
\end{aligned}
$$

and $\chi_{k}^{(m, 8)}=-\phi_{k}^{(m, 8)}$ with $(m+1)$ replaced by $(m-1)$ and vice versa.

Then, at the end of each cycle $m$, the Turing head can be described by $(M=4)$

$$
\begin{aligned}
& K_{10000}^{(m, j)}=0 \\
& K_{20000}^{(m, 8)}=\kappa_{s}^{(m, 8)}\left(\alpha_{1}, \alpha_{2}, \alpha_{3}, \alpha_{4}\right) \\
& K_{30000}^{(m, 8)}=-\kappa^{(m, 8)}\left(\alpha_{1}, \alpha_{2}, \alpha_{3}, \alpha_{4}\right)
\end{aligned}
$$

and the memory cells by

$$
\begin{aligned}
K_{03000}^{(m, 8)} & =\phi_{1}^{(m, 8)} \\
K_{00300}^{(m, 8)} & =\phi_{2}^{(m, 8)} \text { etc. } \\
K_{03300}^{(m, 8)} & =\kappa^{(m, 8)}\left(\alpha_{2}\right) \\
K_{00330}^{(m, 8)} & =\kappa^{(m, 8)}\left(\alpha_{3}\right) \\
K_{00033}^{(m, 8)} & =\kappa^{(m, 8)}\left(\alpha_{4}\right) \\
K_{03030}^{(m, 8)} & =\kappa^{(m, 8)}\left(\alpha_{2}, \alpha_{3}\right) \\
K_{00303}^{(m, 8)} & =\kappa^{(m, 8)}\left(\alpha_{3}, \alpha_{4}\right) \\
K_{03003}^{(m, 8)} & =\kappa^{(m, 8)}\left(\alpha_{2}, \alpha_{3}, \alpha_{4}\right) .
\end{aligned}
$$


The memory pair-correlations are all positive for $m$ even and decay with "step distance", i.e. the number of intermediate rotation and coupling steps to other memory cells (cf. also Fig. 2). The pair correlations between Turing head and the memories are given by

$$
\begin{aligned}
& K_{33000}^{(m, 8)}=\chi_{1}^{(m, 8)} \\
& K_{30300}^{(m, 8)}=\chi_{2}^{(m, 8)} \text { etc. }
\end{aligned}
$$

All the expectation values are strictly periodic in $m$ if $\alpha_{j}=2 \pi / p_{j}$ for all $j=1,2, \cdots, M$ with $p_{j}$ a whole number. The period $p$ is then the smallest even number that has all these $p_{j}$ as factors.

In a similar way one obtains the results for step numbers smaller than $2 M=8$. Generalizations to the situation where the phase angles differ from cycle to cycle are also straight-forward. For example, based on eqs. (39), (40) we find a web of correlations like

$$
K_{33000}^{(m, j)} \cdot K_{03000}^{(m, j)}=K_{30300}^{(m, j)} \cdot K_{00300}^{(m, j)}=K_{30030}^{(m, j)} \cdot K_{00030}^{(m, j)} \quad \text { etc. }
$$

valid for all steps $j$ within any cycle $m$.

\section{$5 \quad$ Reduced descriptions}

\subsection{Turing-head $S$}

The description reduced to the subsystem $S$ is based on the local Bloch-vector $K_{j 0000}, j=$ $1,2,3$ only. Starting from the ground-state, $K_{30000}^{(1,0)}=-1$, this vector is subject to the rotation as given by eq. (19). We see that each controlled NOT operation implies a projection on the 3 -axis $\left(K_{10000}=K_{20000}=0\right)$. The result of eq. (38) for cycle 1 is easily generalized to $M>4$ with $\nu=1,2 \cdots, M$ and $\alpha_{\nu}=\pi / M$. We find

$$
K_{300 \cdots}^{(1,2 M)}=-\cos ^{M}(\pi / M) .
$$

With $\alpha_{\nu}=g t_{2 \nu-1}$ (cf. eq. (21); $2 \nu-1$ is the step number), the quantum-Zeno-effect [13, 14, 15] results within the fixed time $g T_{2 M}=\pi$ (cf. eq. (28)). It is interesting to note that the reduced density matrix (or Bloch-vector) of subsystem $S$ is, at any time $t$, identical with the density matrix of an ensemble of non-interacting spins (all with the same initial state and subject to the same local unitary transformation) but actually measured at each time $T_{2 \nu}, \nu=1,2 \cdots, M$. For each ensemble member the series of measurements constitutes a "decision-tree", with each measurement result given by $K_{30000}^{\prime}= \pm 1$ (see Fig. 2). The ensemble average over these trajectories leads back to the behavior realized here by just one single object! The respective density matrices are identical. This is what one may call quantum parallelism. The interaction with the tape generates a dynamical evolution of the Turing head $S$ equivalent to $2^{M}$ different histories (cf. [16]), clearly an exponential gain. This will only hold, though, as long as no measurements are performed.

\subsection{Turing-tape}

Contrary to the Turing head $S$, the other subsystems are each addressed by unitary transformations only once (within each cycle). Due to the built-in logic the state of subsystem 1 
is strictly anti-correlated with $S$ after preparation step 2 , subsystem 2 is anti-correlated with $S$ after step 4 , and so on. This means that an actual projective measurement performed on these subsystems would reveal also the respective states of $S$. When the transformations are interpreted to happen in parameter-time $T_{2 \mu}$, the subsystems $\mu \neq S$ indeed act as a kind of "memory". They allow delayed measurements on $S$. One may argue that this fact is the origin of the quantum-Zeno-effect discussed in Sect. (5.1): It suffices to be able to measure in order to get the freezing-tendency of measurements ("virtual watchdog"-effect).

Local measurements of the memory cells amounts to the application of a projection-

or transition-operator like $\hat{P}_{01}(\mu)$. As these operators commute among each other (for different $\mu$ ) and with any of the unitary operators not acting on $\mu$, we can postpone these measurements up to one cycle. For $\mu=1$, e.g.,

$$
\begin{aligned}
\hat{U}(S, 4) \hat{U}_{\alpha_{4}}(S) & \ldots \hat{P}_{01}(1) \hat{U}(S, 1) \hat{U}_{\alpha_{1}}(S) \mid \psi^{(m, 0)}>= \\
& \hat{P}_{01}(1) \hat{U}(S, 4) \hat{U}_{\alpha_{4}}(S) \ldots \hat{U}(S, 1) \hat{U}(S)_{\alpha_{1}} \mid \psi^{(m, 0)}>.
\end{aligned}
$$

Let us first restrict ourselves to cycle $m=1$ with its decision tree (Fig. 2). The time order of these measurements (i.e. the measurement process) need not correspond to the time-order, in which the memory cells have been visited by the Turing head: The actual history for the latter (out of the possibilities as shown in Fig. 2) may thus be "realized" even backward in time!

But not only this: The correlation between memory cell 1 and 2, e.g., must, by construction (cf. eq. (35)) and the invariance property $K_{03300}^{(1,8)}=K_{03300}^{(1,4)}$, reflect the correlation between the states of $S$ taken at $T_{2}$ and $T_{4}$, respectively. This is readily verified by comparing our result for $K_{03300}^{(1,8)}$, eq. (39), with $C_{33}^{(S)}$ given by eq. (22) (then a two-time correlation function in the Heisenberg-picture). The fact that $K_{03300}^{(1,8)}$ and $C_{33}^{(S)}$ are identical means, that a measurement of $K_{03300}^{(1,8)}$ can be used to infer the unperturbed $C_{33}^{(S)}\left(T_{2}, T_{4}\right)$. This holds, correspondingly, for $K_{00330}^{(1,8)}$ and $K_{00033}^{(1,8)}$. In this sense time-correlations of the past still "coexist".

As we continue into the cycles $m>1$, the unique identification of tape state and head history is gradually lost; histories become undecidable. The "meaning" of those measurements thus strongly depends on the step- and cycle number. At the end of cycle $m+p$, to be sure, the original situation is restored. The time-parameters $T_{2 \mu}$ labelling those histories are thus defined only modulo $p T_{8}$ (if period $p$ exists).

\section{Special Machines}

\subsection{A "coin-tossing machine"}

For the machine defined by $\left\{\alpha_{\mu}=\pi / 2 ; \mu=1,2,3,4\right\}$ all pair correlations and all one-point expectation-values are zero by the end of cycle $m=1$ (cf. eqs. (39, 38)). The resulting histories all have the same probability and look like those of independent coin tossings at the times $T_{2 \mu}$. As for the "Zeno-machine" $\left\{\alpha_{\mu}=\pi / M ; \mu=1,2, \cdots, M\right\}$, a complete measurement of the tape state at the end of cycle $m=1$ would allow us to reconstruct the history of $S$. The period is $p=4$. 


\subsection{A "cat machine"}

As a next example let us consider the Turing machine defined by $\left\{\alpha_{1}=\pi / 2, \alpha_{2}=\alpha_{3}=\right.$ $\left.\alpha_{4}=0\right\}$. The period is $p=8$, again independent of $M:\left|\psi^{(m, j)}\right\rangle=\left|\psi^{(m+8, j)}\right\rangle$. At the end of any cycle $m$ all memory cells are strictly correlated (cf. eq. (39)). Furthermore,

$$
\mid \psi^{(1,8)}>=\frac{1}{\sqrt{2}}(|11110>-i| 00001>)
$$

is found to be a so-called cat-state, for which the decision tree of Fig. 2 collapses to two histories only, (1111) and (0000), respectively. Moreover $\left|\psi^{(5,8)}\right\rangle$ is a different one. As a process the built-up of these cat-states is thus quite simple. While cat states are reduced to product states by the decay (measurement) of any individual subsystem, all the memory pair correlations discussed here remain intact as long as the decaying subsystem is not part of that very pair.

\subsection{Large-scale predictability}

For $m=100<p$ and $M+1=10$ we would have roughly $m 2^{M} \approx 5 \cdot 10^{4}$ transformations in a $2^{M+1} \approx 1000$-dimensional Hilbert-space; nevertheless, the calculation of these expectation values would scale, at most, linearly with $M$, independent of $m$ ! This indicates that simulations even of large networks could become feasible based on such rules. Of course, the number of expectation values increases exponentially with the system size $M+1$.

\section{Conclusions}

We have discussed the dynamics of a special quantum network, which combines quantummechanical and classical features: The quantum-mechanical variables consist of a "Turing head" (pseudospin $S$ ) and a "Turing tape" ( $M$ memory spins). Classical variables are the phenomenological Hamilton-parameters, which are switched externally to generate discrete unitary transformations. The machine behavior is defined by its initial state and the phase angles $\alpha_{\mu}$ specifying those transformations.

This switching can be visualized as being induced by the Turing head performing predetermined cycles over $2 M$ Turing head positions. Correlations in terms of multi-point expectation values are built up in this process. Time defines the order of non-commuting operations and quantitatively controls transformation parameters.

The structure of these correlations may be attributed to the notorious "holistic nature" of quantum mechanics. Nevertheless, this built-up follows a strict logic; the type of admissible manipulations (rotations) is severely constrained in all but the simplest 2-level-space; this observation certainly applies to our present $2^{M+1}$-level-model. Additional constraints are built in by the selection of transformations which are actually implemented. Here they relate to the fact that the multi-levels actually refer to $M+1$ subsystems. These constraints are reflected by the spatio-temporal pattern of correlations.

There is probably good news and there is bad news as far as the consequences are concerned: The bad news is that the implementation of specific processes is much more 
constrained in the quantum regime than in the macroscopic world; this makes experimental progress in quantum computation depressively slow. The good news could be that, eventually, only constrained systems can make up a useful machinery; systems with large, unrestricted state spaces (like a free gas) are "useless". The constraints are something like fixed axles, wheels, and connecting rods in classical mechanics. Under fairly moderate conditions those correlations and the correlation between correlations should constitute a machine behavior. Rather than enforcing some specific behavior defined by abstract algorithms we might be better off trying to exploit the experimental repertoire of real quantum networks.

\section{Acknowledgments and figure captions}

We thank C. Granzow, A. Otte and R. Wawer for fruitful discussions.

Fig. 1 Quantum Turing machine $(M=4)$.

The circular Turing tape consists of $\mu=1,2, \cdots, M$ memory cells (position $2 \mu$ ) separated by empty cells (position $2 \mu-1$ ). The Turing head moves clockwise thus initiating a local (position-index odd) or a pair transformation, respectively (position index even).

Fig. 2 Alternative histories.

a. Decision tree with respect to step number $2 \mu=2,4,6,8$, as realized in an ensemble of non-interacting spins $S$ under the series of local transformations $\hat{U}_{\alpha_{\mu}}(S)$, but with immediate actual measurements (replacing $\hat{U}(S, \mu)$ of our Turing machine) at times $T_{2 \mu}$.

b. For the single Turing machine all the possible histories are yet undecided and associated with the states of the Turing tape as given.

\section{References}

[1] A. Ekert and R. Jozsa, Rev. Mod. Phys. 68, 733 (1996)

[2] A. Barenco et. al., Phys. Rev. A 52, 3457 (1995)

[3] J. I. Cirac and P. Zoller, Phys. Rev. Lett. 74, 4091 (1995)

[4] P. Domokos, J. M. Raimond, M. Brune, and S. Haroche, Phys. Rev. A 52, 3554 (1995)

[5] N. A. Gershenfeld and I. L. Chuang, Science 275, 350 (1997)

[6] A. Shnirman, G. Schön, and Z. Hermon, Phys. Rev. Lett. 79, 2371 (1997)

[7] G. Mahler and V. A. Weberruß, Quantum Networks: Dynamics of Open Nanostructures, Springer Berlin, New York 1995; 2nd revised edition 1998

[8] M. Ferrero and E. Santos, Found. Phys. 27, 765 (1997)

[9] J. Schlienz and G. Mahler, Phys. Rev. A 52, 4396 (1995) 
[10] D. Deutsch, Proc. Roy. Soc. A 400, 97 (1985)

[11] J. P. Paz and G. Mahler, Phys. Rev. Lett. 71, 3235 (1993)

[12] K. Obermayer, W. G. Teich, and G. Mahler, Phys. Rev. B 37, 8111 (1988)

[13] B. Misra and E. C. G. Sudershan, J. Math. Phys. 18, 756 (1977)

[14] P. Knight, Nature 344, 493 (1990)

[15] W. M. Itano, D. J. Heinzen, J. J. Bollinger and D. J. Wineland, Phys. Rev. A 41, 2295 (1990)

[16] R. Omnes, Rev. Mod. Phys. 64, 339 (1992) 


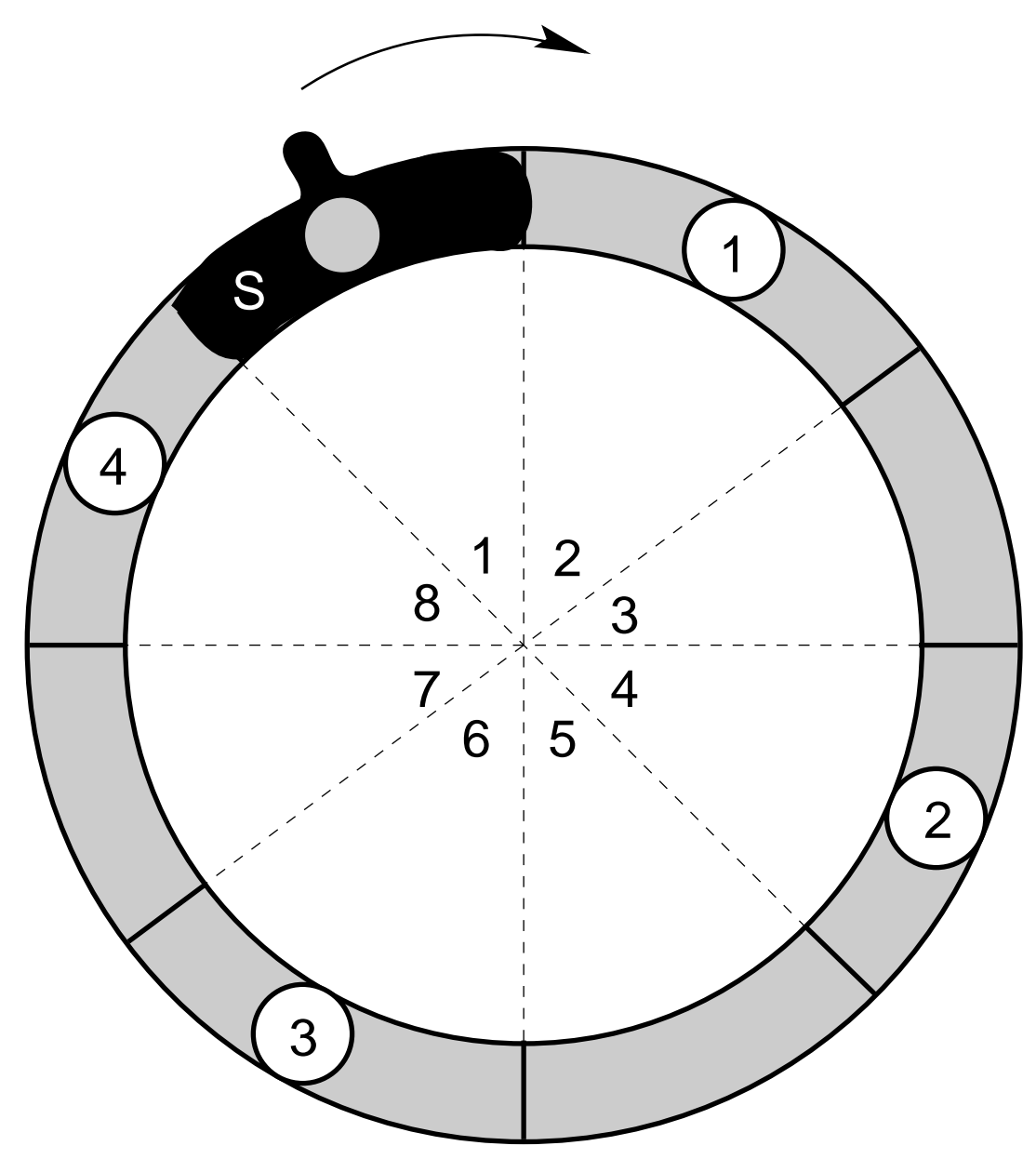




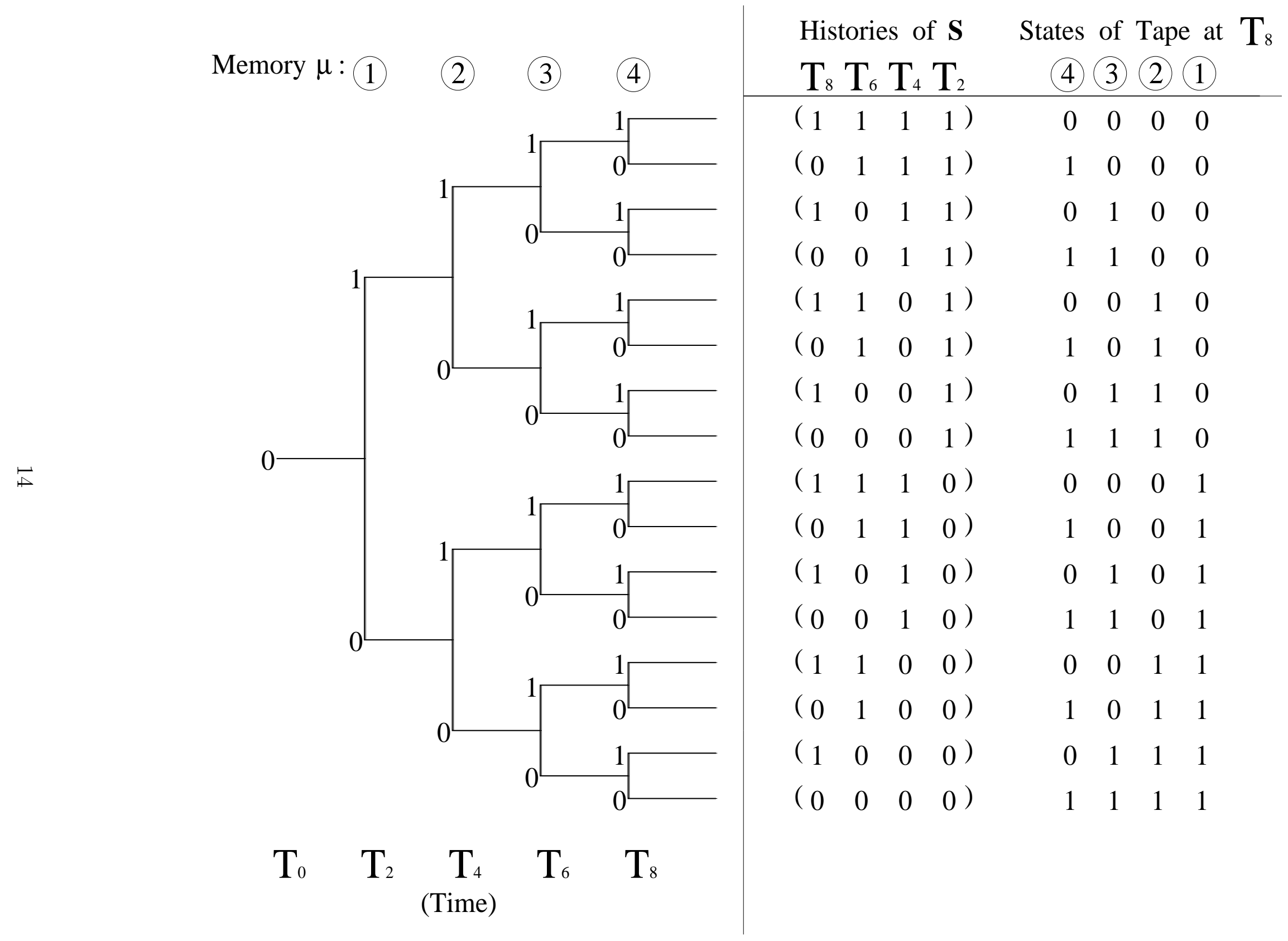

